\title{
ESPECTROFOTOMETRIA MULTICANAL E ARRANJOS DE FOTODIODOS
}

\author{
Ivo M. Raimundo Jr. e Celio Pasquini \\ Departamento de Química Analítica - Instituto de Química - UNICAMP - CP 6154 - 13083-970 - Campinas - SP
}

Recebido em 20/3/96; aceito em 19/6/96

\begin{abstract}
MULTICHANNEL SPECTROPHOTOMETRY AND PHOTODIODE ARRAYS. This paper describes some aspects of multichannel spectrophotometry, principles of photodiode arrays and their applications in Analytical Chemistry.
\end{abstract}

Keywords: photodiode array detectors; multichannel spectrophotometry; instrumentation.

\section{ESPECTROFOTOMETRIA MULTICANAL}

Os sistemas ópticos empregados em espectroscopia podem ser classificados, dentre outras maneiras, como multiplexados e não-multiplexados.

Os instrumentos multiplexados empregam um único detector que recebe informações simultâneas codificadas segundo um determinado padrão, que são posteriormente transformadas, através de métodos matemáticos, em informações espectrais. Estes instrumentos podem, ainda, utilizar sistemas dispersivos ou não-dispersivos, cujos exemplos mais significativos são os espectrofotômetros com transformadas de Hadamard $^{1}$ e de Fourier $^{2}$, respectivamente.

Os instrumentos não-multiplexados podem, entretanto, empregar um ou vários detectores ${ }^{3,4}$. Os que empregam apenas um detector são chamados monocanais ou temporais e monitoram de forma sequencial as intensidades de radiação do espectro eletromagnético. Aqueles que empregam vários detectores são chamados multicanais ou espaciais, monitorando simultaneamente vários comprimentos de onda. Os instrumentos não-multiplexados também podem ser dispersivos, empregando, via de regra, uma rede de difração, e não-dispersivos, empregando, por exemplo, filtros ou diodos emissores de luz. Atualmente, os sistemas dispersivos são os mais encontrados, sendo que os espectrofotômetros monocanais normalmente usam uma rede de difração rotatória (movida manual ou automaticamente) e como detector uma fotomultiplicadora (PMT, "photomultiplier tube") fixada na fenda de saída do monocromador. Por sua vez, os instrumentos multicanais geralmente têm um monocromador fixo, podendo empregar como detectores filmes fotográficos, arranjo de fotomultiplicadoras, dispositivos de transferência de carga (CTD, "Charge Transfer Devices") e arranjos de fotodiodos (PDA, "Photodiode Array").

Uma das vantagens que podem ser obtidas com instrumentos multicanais está diretamente relacionada com o tempo de análise e com o ruído associado ao sinal medido. A simultaneidade na aquisição de dados pode resultar, em comparação com um instrumento monocanal, em uma análise mais rápida se a razão sinal/ruído for mantida constante ou em um aumento na razão sinal/ruído se o tempo de análise for fixado ${ }^{5}$. Isto pode ser facilmente compreendido ao se fazer uma analogia com a pesagem de vários objetos em várias balanças, onde a massa de um objeto está relacionada com a intensidade de um comprimento de onda medido e cada balança associada a cada detector $^{6}$. Supondo que existam quatro objetos para serem pesados em quatro balanças, dois procedimentos podem ser adotados. O primeiro consiste na pesagem simultânea destes quatro objetos em cada uma das quatro balanças, o que leva a uma determinação das massas em um tempo quatro vezes menor que com o uso de uma única balança. $\mathrm{O}$ segundo procedimento consiste na pesagem de cada um dos objetos nas quatro diferentes balanças, no mesmo tempo que seria necessário para pesá-los em uma única balança. Desta forma, cada objeto é pesado quatro vezes e a estimativa do desvio padrão relativo na massa de cada objeto é reduzida à metade $(\sqrt{4})^{7}$. Da mesma forma, quando comparado com um espectrofotômetro convencional, um multicanal disposto com $n$ detectores pode, teoricamente, obter um espectro em um tempo $n$ vezes menor ou com um aumento na razão sinal/ruído de aproximadamente $\sqrt{n}$.

Os instrumentos multicanais podem ser desenvolvidos com o uso de arranjos de fotomultiplicadoras como, por exemplo, os encontrados nos espectrofotômetros de emissão atômica em plasma acoplado indutivamente (ICP-AES). Estes instrumentos são classificados como espectrômetros de leitura direta ${ }^{4}$, sendo que as fotomultiplicadoras empregadas, a despeito de suas vantagens referentes a sensibilidade, faixa dinâmica e tempo de resposta, trazem alguns inconvenientes incompatíveis com um detector multicanal verdadeiro. Embora miniaturizadas, estas fotomultiplicadoras podem ser dispostas nestes espectrofotômetros a uma distância mínima que ainda requer uma grande dispersão da radiação eletromagnética, o que inevitavelmente limita o número de canais do instrumento. Desta forma, os espectrofotômetros de leitura direta são fabricados para aplicações específicas, monitorando certos comprimentos de onda e sendo, portanto, pouco flexíveis ${ }^{4,8,9}$.

Os espectrofotômetros com arranjos lineares de fotodiodos trouxeram, nos últimos quinze anos, uma nova dimensão para a espectrofotometria, principalmente a UV-visível, quando comparados com os espectrofotômetros com banco de fotomultiplicadoras ou com redes de difração (ou espelhos) móveis. A figura 1 mostra os esquemas de espectrofotômetros com PDA e com rede de difração móvel, salientando suas principais

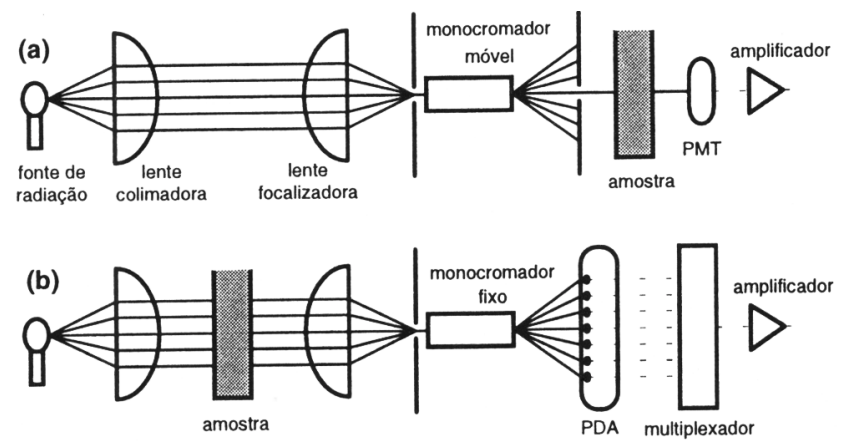

Figura 1. Esquema de espectrofotômetros convencional com rede de difração móvel (a) e multicanal com arranjo de fotodiodos (b). 
diferenças ${ }^{10}$. O espectrofotômetro de arranjo de diodos emprega um sistema de óptica reversa ${ }^{11}$, isto é, a rede de difração é colocada entre a amostra e o detector e não entre a fonte de radiação e a amostra. Assim sendo, no PDA a radiação policromática incide sobre a amostra e é, então, dispersa em um monocromador fixo em diferentes comprimentos de onda que são monitorados simultaneamente pelos diodos do arranjo. Esta configuração com monocromador fixo traz algumas vantagens, possibilitando ao espectrofotômetro PDA tanto adquirir um espectro sem distorções e em poucos milisegundos como medir os comprimentos de onda com alta repetibilidade, pois o tempo de varredura não é determinado pelo movimento da rede de difração e a repetibilidade é limitada apenas pela geometria do detector, respectivamente. Entretanto, um espectrofotômetro PDA pode estar mais sujeito aos problemas provenientes tanto da radiação espúria, que não pode ser eliminada por meio de filtros, como das estabilidades da fonte e/ou do detector, que não podem ser facilmente compensadas com um sistema de duplo feixe, como feito nos espectrofotômetros convencionais. Por outro lado, os problemas associados à incidência de radiação policromática sobre a amostra, tais como aquecimento e fotodegradação, podem ser minimizados com o uso de obturador e/ou filtros apropriados.

Portanto, por estes aspectos, a aplicação de um espectrofotômetro multicanal com um arranjo de fotodiodos é particularmente recomendada no estudo de sistemas com sinais transientes (como FIA e HPLC) e na diferenciação de compostos que absorvem em comprimentos de onda bastante próximos. Todavia, é importante salientar que nos casos onde o tempo para a obtenção de um espectro não é parâmetro crítico, o desempenho de um espectrofotômetro convencional com rede de difração móvel é, ainda, melhor que um com PDA, devido à maior sensibilidade apresentada por uma fotomultiplicadora.

\section{DETECTORES DE ARRANJO DE FOTODIODOS}

Um detector de arranjo de fotodiodos consiste em um circuito integrado único que possui um sensor de radiação, um elemento de armazenamento de carga e um elemento de leitura. O arranjo de diodos é fabricado em um único cristal de silício, onde cada diodo atua simultaneamente como transdutor de intensidade luminosa/carga elétrica e como elemento de armazenamento de carga.

O PDA é preparado pela oxidação da superfície de um substrato semicondutor* do tipo-n, produzindo uma camada de óxido de silício com espessura de $0,4-3,0 \mu \mathrm{m}^{12,13}$. Pequenas janelas são então abertas na camada de óxido por um processo de fotolitografia, onde são formadas as junções pn, através da difusão de um semicondutor do tipo-p. Esta camada difusa de silício tipo-p pode ter uma espessura de 1,2 - 1,5 $\mu \mathrm{m}$. A figura 2 mostra um esquema do cristal de silício e da geometria do sensor. Os arranjos com esta geometria $(2,5 \mathrm{~mm}$ de altura e espaçamento centro a centro de $25 \mu \mathrm{m}$ ) são específicos para fins espectroscópicos pois possuem as mesmas proporções (100:1) de uma fenda de saída de um monocromador convencional.

$\mathrm{Na}$ junção pn, os transportadores de carga majoritários de ambos os semicondutores migram em sentidos opostos, isto

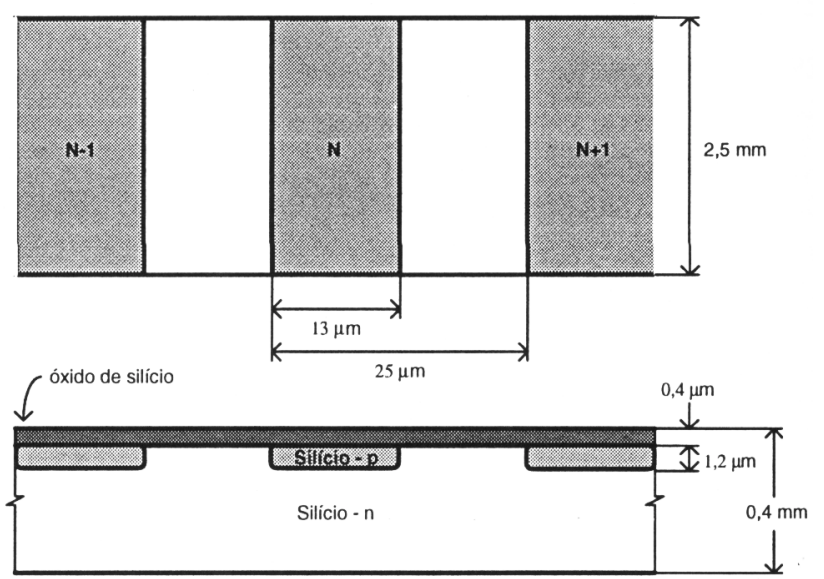

Figura 2. Esquema de um arranjo linear de fotodiodos.

é, os elétrons migram do semicondutor tipo-n para o -p e as lacunas migram do semicondutor tipo-p para o $-n$. Com este processo de difusão, ocorre a formação de um potencial na interface cujo campo elétrico se opõe à difusão adicional dos transportadores majoritários através da junção. Desta forma, na região adjacente à junção, o semicondutor tipo-n fica positivamente carregado em relação ao tipo-p (Fig. 3a). Na região contígua à junção pn é criada uma zona de recombinação, conhecida como zona de depleção, onde um elétron tem maior probabilidade de preencher uma lacuna da banda de valência. Nesta região, existem poucos transportadores e, portanto, a resistência é alta. O dispositivo assim construído com a junção dos materiais tipo-n e -p é conhecido como diodo semicondutor. Em um PDA, a junção pn é polarizada reversamente, aumentando a barreira de energia que os transportadores de carga precisam atravessar devido ao aumento da zona de depleção (Fig. 3b), permitindo, assim, que cada diodo do arranjo se comporte como um minúsculo capacitor ${ }^{12,14}$. Os fótons

(a)
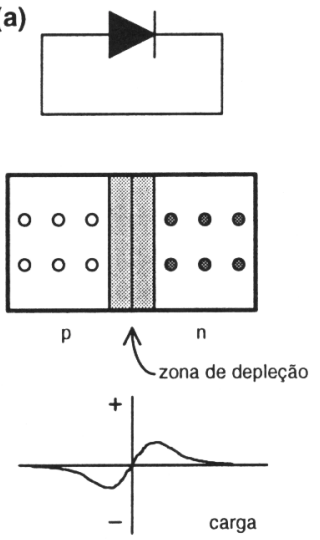

Figura 3. Influência da polarização sobre a zona de depleção: sem polarização $(\boldsymbol{a})$ e com polarização reversa $(\boldsymbol{b})$.

\footnotetext{
* Um material é semicondutor quando possui a banda de valência totalmente preenchida com elétrons, cuja diferença de energia em relação à banda de condução ("band gap") não é muito grande. Em outras palavras, o semicondutor é capaz de transferir um elétron da banda de valência para a banda de condução às custas de uma quantidade de energia muito pequena. Um semicondutor intrínseco é um material puro no qual a energia térmica é suficiente para promover um elétron da banda de valência para a banda de condução. Este processo cria um centro positivo na banda de valência, chamado lacuna ("hole"), capaz de se mover através desta banda. Em um semicondutor, tanto os elétrons como as lacunas, chamados de transportadores, dão origem à corrente elétrica. Um semicondutor extrínseco possui defeitos em seu retículo cristalino, provocado através de um processo de dopagem. Se o semicondutor intrínseco é dopado com uma "impureza química" que possui mais elétrons na banda de valência que o substrato, obtem-se um semicondutor do tipo-n (elétrons são os transportadores majoritários). Caso contrário, se o dopante possui menos elétrons na banda de valência que o semicondutor intrínseco, obtem-se um semicondutor tipo-p (lacunas são os transportadores majoritários). É importante notar que os semicondutores tipo-n e -p não são carregados negativa e positivamente, como pode ser induzido a pensar ${ }^{12}$.
} 
absorvidos tanto pela região - $p$ como pela $-n$ podem produzir lacunas e elétrons, se possuírem energia suficiente para isto. Estas lacunas e estes elétrons são atraídos, respectivamente, para os polos negativo e positivo do diodo reversamente polarizado, isto é, as lacunas migram para a região -p e os elétrons para a região $-n$. Os fótons absorvidos entre duas ilhas de semicondutor tipo-p (isto é, absorvidos pela região -n) produzem cargas que são proporcionalmente divididas entre os diodos adjacentes. A figura 4 mostra a função de resposta de um diodo do arranjo, devido à radiação inicidente sobre a região A. Para este diodo, $52 \%$ da carga gerada é proveniente da região $-\mathrm{p}$, $36 \%$ da região -n e $12 \%$ é dividida entre os diodos adjacentes ${ }^{14}$. Portanto, a absorção de fótons descarrega o minúsculo capacitor formado pelo diodo reversamente polarizado. Este processo também ocorre devido à formação de cargas geradas termicamente, conhecidas como corrente de escuro ("dark current"). Desta forma, em muitas aplicações, principalmente naquelas onde existe a necessidade de longos períodos de exposição, o arranjo de diodos deve ser resfriado. O resfriamento é normalmente efetuado com nitrogênio líquido ou com elementos tipo Peltier, sendo que a corrente de escuro diminui à metade para uma diminuição de $7-9{ }^{\circ} \mathrm{C}$ na temperatura ${ }^{13,14}$.

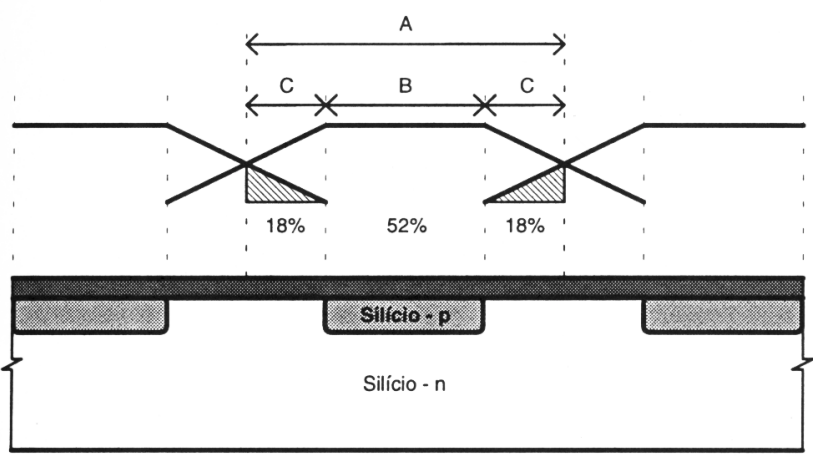

Figura 4. Função de resposta idealizada de um diodo do arranjo devido à radiação incidente sobre $25 \mu \mathrm{m}$ a partir do centro do diodo $(A)$, diodo (região - $p$ ) de $13 \mu \mathrm{m}(B)$ e região - $n$ da matriz de $6 \mu \mathrm{m}(C)$.

Logo, mantida a corrente de escuro em níveis desprezíveis, o capacitor formado pelo diodo descarrega proporcionalmente à potência de radiação luminosa que nele incide e ao tempo de exposição à esta radiação. Além disto, a sensibilidade do diodo é dependente do comprimento de onda, como pode ser verificado na figura 5 .

Os arranjos de diodos podem ser encontrados com 128 até 4096 elementos, sendo os mais comuns os de 512 e de 1024.

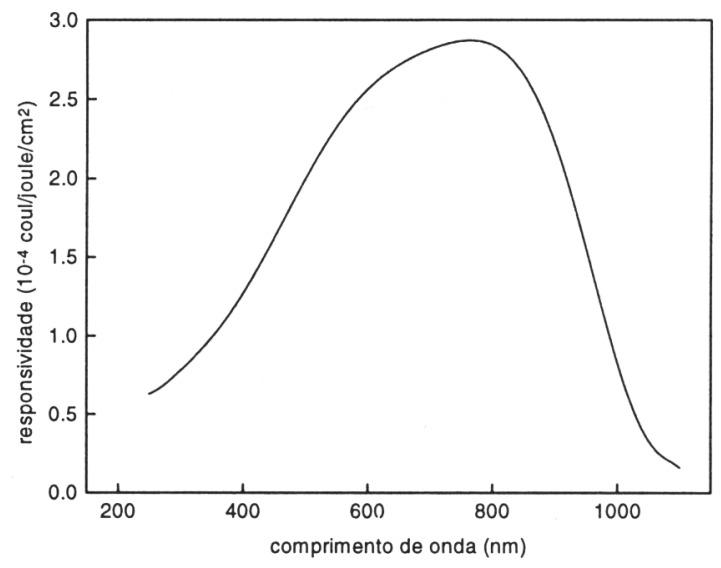

Figura 5. Resposta espectral de um arranjo de diodos EGG-Reticon $S$, com janela de quartzo ${ }^{15}$.
Embora a detecção da radiação seja simultânea, o processo de leitura de cada diodo é sequencial. A figura 6 mostra um esquema simplificado do circuito de um PDA.

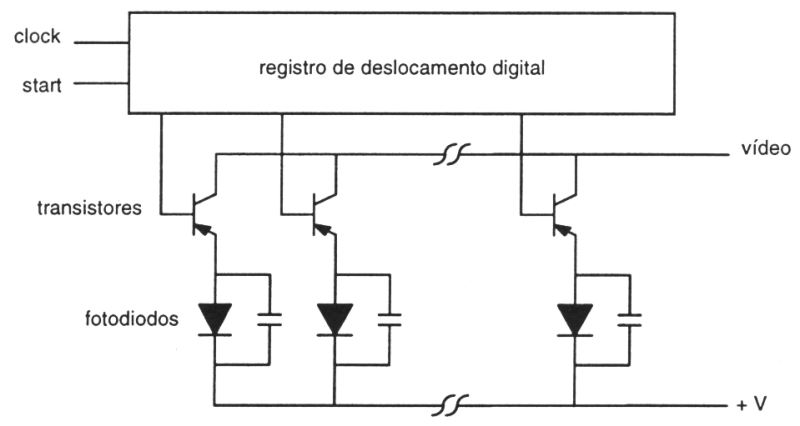

Figura 6. Esquema simplificado de um arranjo linear de fotodiodos.

É importante salientar que o capacitor mostrado nesta figura (ligado em paralelo com o diodo) não é um componente adicional do circuito mas sim uma representação da capacitância da junção pn. Cada fotodiodo do arranjo é conectado a uma chave-transistor (FET, "field effect transistor"), que é controlada por um único sinal que é deslocado através de um registro digital ("digital shift register"). Um pulso de "start" (início de varredura) é usado para iniciar o processo de leitura. Após este pulso, os transistores FET são endereçados sequencialmente a cada pulso de "clock". Quando um transistor é endereçado, o diodo a ele associado é totalmente recarregado ao seu potencial de polarização reversa e a corrente que flui através do diodo e do transistor aparece na saída de vídeo. Este sinal analógico é amplificado, extraído por um circuito amostrador/retentor e, então, apresentado a um conversor analógico-digital. A frequência de "clock" empregada na leitura dos diodos pode variar de $36 \mathrm{kHz}(28 \mu \mathrm{s} /$ diodo $)$ até $2 \mathrm{MHz}(0,5 \mu \mathrm{s} / \text { diodo })^{5,14}$. O tempo de integração é definido como o intervalo entre dois pulsos de "start" de duas varreduras consecutivas ${ }^{14}$, sendo o mesmo para todos os diodos pois, uma vez iniciado o processo de leitura, o arranjo normalmente deve ser varrido sequencial e totalmente. Entretanto, como o PDA necessita de um intervalo de tempo $\Delta \mathrm{t}$ para ler cada um dos $n$ sucessivos elementos, o $n$ 'ésimo diodo do arranjo terá integrado a radiação por um mesmo intervalo de tempo, mas em um momento que difere do primeiro diodo em $n . \Delta \mathrm{t}$. A figura 7 ilustra este detalhe de um arranjo linear de fotodiodos. Embora esta diferença de tempo seja desprezível em muitos casos, como por exemplo em HPLC e FIA, em algumas aplicações, como no estudo de reações rápidas, o erro introduzido pode causar uma distorção temporal significativa ${ }^{14}$.

O desempenho global de um espectrofotômetro com arranjo de fotodiodos é determinado, em grande parte, pelas características

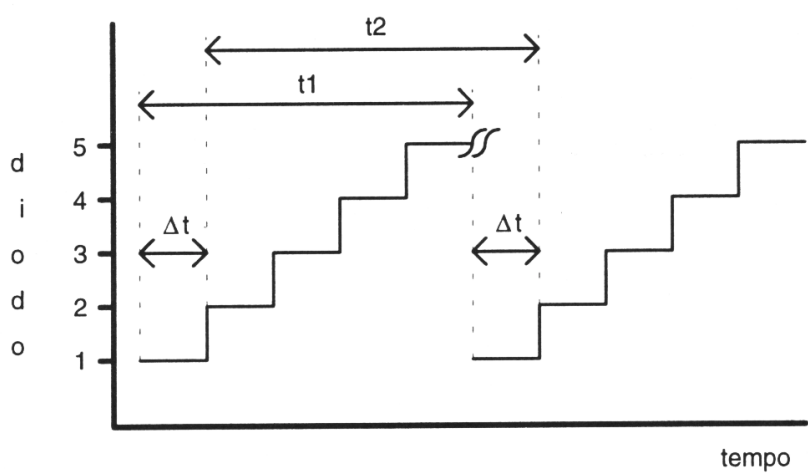

Figura 7. Sequência de leitura de um PDA: t1 e t2 são iguais e correspondem aos tempos de integração dos diodos 1 e 2, respectivamente. 
do detector, tais como faixa de resposta espectral, exatidão e precisão nas medidas de comprimento de onda e intensidade luminosa, resolução, sensibilidade, razão sinal/ruído e faixa dinâmica. A tabela 1 destaca algumas destas características (e outros parâmetros relacionados) de um arranjo de diodos e de uma fotomultiplicadora. Esta comparação está sendo feita pois, de certa forma, o desenvolvimento do espectrofotômetro PDA é consequência da necessidade de obtenção de espectros em curtos intervalos de tempo, os quais não podem ser facilmente conseguidos com um espectrofotômetro com rede de difração móvel que emprega uma PMT como detector. É interessante notar que embora a eficiência quântica de uma fotomultiplicadora seja menor que a de um diodo, a sua sensibilidade é muito maior, devido ao alto ganho que possui. No exemplo citado na tabela 1, a sensibilidade da fotomultiplicadora é de $0,067 \mathrm{~A} \mathrm{~W}^{-1}$, mas com o ganho de $6 \times 10^{5}$, o valor de $4,0 \times 10^{4}$ é obtido, tornando-a mais sensível que o diodo ${ }^{17}$.

Tabela 1. Comparação entre os desempenhos de um arranjo de diodos (PDA) e de uma fotomultiplicadora (PMT).

\begin{tabular}{ccc}
\hline Parâmetro & PDA & PMT \\
\hline resposta espectral $(\mathrm{nm})^{\mathbf{a}}$ & $200-1000$ & $200-650$ \\
eficiência quântica $(\%)$ & $40-80$ & $10-20$ \\
sensibilidade $\left(\mathrm{A} \mathrm{W} \mathrm{W}^{-1}\right)^{\mathbf{b}}$ & 0,45 & $4,0 \times 10^{4}$ \\
faixa dinâmica & $10^{3}-10^{4}$ & $10^{5}-10^{6}$ \\
corrente de escuro $\left(\mathrm{e}^{-} \mathrm{s}^{-1}\right)^{\mathbf{a}}$ & $624(193 \mathrm{~K})$ & $3(253 \mathrm{~K})$ \\
\hline
\end{tabular}

(a) PDA Reticon 1024S x PMT Galileo Eletro-Optics ${ }^{15}$.

(b) PDA Reticon x PMT RCA C $31034^{16}$.

A faixa dinâmica de um arranjo de diodos pode ser medida de diferentes maneiras, sendo duas as mais importantes ${ }^{12,14,17}$. Uma delas, definida como a razão entre o maior e o menor sinal obtidos em um mesmo intervalo de tempo e expressa na tabela 1, está relacionada com a resposta de apenas um diodo, podendo ser empregada para comparação com a resposta de uma fotomultiplicadora. Enquanto que para a PMT esta faixa é limitada pela corrente de escuro, para o PDA o limite é geralmente dado pelo conversor analógico/digital. Por exemplo, assumindo uma incerteza de \pm 1 bit, para um A/D de 14 bits a razão máxima é de 1:8192, isto é, o maior sinal dividido pelo dobro do ruído de conversão ${ }^{17}$. A outra forma de se medir a faixa dinâmica, chamada de faixa dinâmica intra-espectral simultânea ou intra-cênica, é definida como a razão entre o maior e o menor sinal obtidos simultaneamente em uma mesma varredura do arranjo ${ }^{13}$. Esta faixa é geralmente menor que a primeira, sendo limitada pela corrente de escuro e pela reflexão de luz que ocorre entre a janela que cobre o arranjo de diodos e a sua superfície ${ }^{13}$.

A exatidão na medida de comprimento de onda por um arranjo de diodos, determinada pela geometria do detector e pela dispersão linear recíproca do monocromador, é limitada, assumindo a estabilidade da fonte de radiação, por vibrações e expansão térmica dos componentes ópticos do sistema. Esta exatidão, equivalente ao produto do espaçamento entre os diodos (ou largura do diodo, tipicamente $25 \mu \mathrm{m}$ ) e a dispersão linear recíproca $\left(\mathrm{nm} \mathrm{mm}^{-1}\right)$, é geralmente melhor que a exatidão de um espectrofotômetro de varredura, que é limitada por suas partes mecânicas móveis ${ }^{13,14}$, como discutido anteriormente.

Assim como a exatidão, a resolução de um PDA também é determinada pela largura do diodo e pela dispersão linear recíproca do monocromador, sendo muitas vezes definida, de maneira incorreta, como sendo o produto destes dois parâmetros. $\mathrm{Na}$ verdade, como o perfil de resposta de um arranjo de diodos é trapezoidal e não retangular (Fig. 4), provocando uma sobreposição parcial de informações espectrais, a resolução é piorada por um fator de $2,0-2,5^{5,13}$. Apesar deste fato, a resolução obtida com um espectrofotômetro de arranjo linear de fotodiodos é ainda bastante adequada para um grande número de aplicações analíticas.

Finalmente, é importante salientar que pela própria natureza de um PDA, que é capaz de gerar um número relativamente grande de dados num intervalo de tempo bastante pequeno, um espectrofotômetro multicanal com este detector necessita ser controlado por um microcomputador com desempenho razoável. Portanto, talvez tenha sido por esta necessidade que apenas na década de 80 tenham surgido os primeiros instrumentos comerciais.

\section{DESENVOLVIMENTO E APLICAÇÕES ANALÍTICAS DE ESPECTROFOTÔMETROS COM ARRANJO LINEAR DE FOTODIODOS}

Os espectrofotômetros que possuem PDAs como detectores são, sem dúvida alguma, instrumentos multicanais muito acessíveis e, portanto, bastante empregados nos dias de hoje. A utilização de arranjos de fotodiodos em espectroscopia teve início há cerca de 25 anos, sendo que um dos primeiros espectrofotômetros comerciais foi fabricado pela HewlettPackard (HP 8450A) no final da década de $70^{5}$.

No início do desenvolvimento de detectores multicanais (ou de imagem), alguns dos principais objetivos estavam pautados pela necessidade de substituição do filme fotográfico por um sistema de detecção eletrônico que possibilitasse a aquisição e interpretação dos dados de maneira simples e eficiente, aliando a conveniência da detecção simultânea obtida com o filme. Prova disto está nos primeiros trabalhos publicados que empregam arranjos de diodos como detectores em espectroscopia de emissão atômica ${ }^{18-21^{* *}}$. Após uma primeira experiência na construção e aplicação de um arranjo de vinte diodos na determinação simultânea de cálcio e bário por espectrometria emissão atômica em chama ${ }^{18}$, Boumans verificou que havia a necessidade de cooperação entre especialistas nas áreas de espectroscopia e eletrônica para o desenvolvimento de um arranjo de diodos com características próprias para ser usado como detector em espectrômetros ${ }^{19}$. Paralelamente, Codding e Horlick empregavam um arranjo de 256 fotodiodos (Reticon RL 256/128) como detector tanto em espectrografia para a identificação de vários elementos ${ }^{20}$ como em espectrofotometria de emissão e absorção atômica para a determinação de potássio, rubídio e cálcio ${ }^{21}$, sendo que os sinais gerados pelo arranjo podiam ser digitalizados e armazenados em um pequeno computador ou mostrados no monitor de vídeo de um osciloscópio. Desde então, os sistemas de detecção e os espectrofotômetros com arranjos de diodos têm sido largamente difundidos e cada vez mais aplicados em várias áreas da Química Analítica, tanto nas que empregam sistemas hidrodinâmicos, tais como FIA e HPLC, como naquelas que empregam sistemas discretos e necessitam apenas da detecção multicanal.

Os primeiros trabalhos empregando arranjos de fotodiodos como detectores em HPLC foram apresentados, em 1976, por

\footnotetext{
** Outros detectores de imagem, como citado anteriormente, podem ser empregados em espectroscopia. Na verdade, estes detectores têm sido usados desde 1949, quando Agnew e colab. ${ }^{22}$ e Benn e colab. ${ }^{23}$ empregaram um tubo Orticon de imagem de televisão no estudo espectroscópico de espécies transientes produzidas por combustão. Outros tipos, tais como tubos Silício-Vidicon e Vidicon Intensificado, também podem ser empregados como detectores de imagem mas o são em menor escala e aparecem esporadicamente na literatura ${ }^{24}$. Uma descrição detalhada de todos estes detectores pode ser encontrada em Busch e Busch ${ }^{25}$.
} 
Dessy e colaboradores ${ }^{26,27}$ e Milano e colaboradores ${ }^{28}$. No início da década de 80 , quando já existiam espectrofotômetros PDA disponíveis comercialmente, os sistemas HPLC-PDA eram discutidos e suas potencialidades evidenciadas ${ }^{29,30}$. Estes sistemas têm experimentado, desde então, uma evolução significativa, que pode ser verificada pela fabricação de espectrofotômetros comerciais com "softwares" dedicados à HPLC. Os detectores PDA possibilitaram o desenvolvimento de métodos mais sensíveis e seletivos, sendo amplamente aplicados às áreas industrial, farmacêutica e clínica ${ }^{10,11}$. A obtenção de um espectro para cada tempo de retenção permite não só uma verificação mais completa a respeito da pureza do pico cromatográfico e consequentemente da eficiência da separação ${ }^{10}$ como também uma análise mais rápida, pois em uma única eluição todos os cromóforos podem, em tese, ser observados ${ }^{31}$. Além disto, espécies mal separadas cromatograficamente podem ser eventualmente determinadas caso não exista uma grande sobreposição espectral ${ }^{10,31}$. Estas vantagens têm sido frequentemente aproveitadas nos métodos descritos na literatura ${ }^{32-34}$.

Os sistemas PDA para análise por injeção em fluxo, no início, não tiveram o mesmo desenvolvimento que os similares para HPLC. A primeira aplicação de um PDA como detector em sistemas de análise em fluxo foi proposta em 1979 por Anderson e colaboradores para a determinação simultânea de níquel e cobalto ${ }^{35}$. Os autores salientaram ainda que poucos esforços tinham sido envidados no desenvolvimento de instrumentos para este fim, devido à dificuldade de serem encontrados reagentes que formassem complexos com vários metais, cujos espectros de absorção fossem suficientemente diferentes para identificá-los em uma mistura. Em 1986, Lázaro e colaboradores, em artigo de revisão, afirmaram que a associação FIAPDA era "interessante, a priori", em decorrência das similaridades existentes entre os sistemas FIA e HPLC, podendo ser justificada principalmente nos casos onde a determinação simultânea era possível em função das diferenças espectrais entre duas espécies ${ }^{11}$. No mesmo ano, estes autores publicaram o primeiro trabalho de determinação simultânea em sistemas FIA$\mathrm{PDA}^{36}$, descrevendo um método para a determinação simultânea de cobre (II) e ferro (II) ou ferro (III) e outro para a determinação de nitrito em uma ampla faixa de concentração. Enfatizaram ainda que, tendo em vista as determinações simultâneas, o desenvolvimento natural da análise por injeção em fluxo deveria passar pela sua associação com detectores de varredura rápida. Atualmente, os detectores multicanais, em especial os arranjos de fotodiodos, têm sido largamente utilizados em sistemas FIA, possibilitando que determinações simultâneas possam ser efetuadas tanto nos casos onde existam diferenças significativas nos espectros de absorção das espécies ${ }^{37}$ como naqueles onde a sobreposição é significativa, que é resolvida pelo uso de métodos derivativos ${ }^{38}$, mínimos quadrados parciais $^{39,40}$ ou regressão linear múltipla ${ }^{41}$. Os sistemas FIAPDA têm grande potencial para explorar os gradientes que podem ser gerados em análise por injeção em fluxo ${ }^{42,43}$, possibilitando a obtenção de superfícies tridimensionais (Absorbância $\mathrm{x}$ comprimento de onda $\mathrm{x}$ tempo), cuja aplicação foi denominada "Flow Chemography" 44 . Além disto, o uso de detectores multicanais em sistemas FIA possibilita que sinais espúreos devido ao efeito Schlieren sejam compensados, aumentando também a razão sinal/ruído ${ }^{45}$.

Os espectrofotômetros com arranjo de fotodiodos disponíveis comercialmente são, em geral, de custo relativamente alto e, algumas vezes, têm a característica peculiar de incorporarem aplicações desnecessárias e, ao mesmo tempo, não poderem ser aplicados em sistemas mais simples de interesse do usuário. Este problema ocorre porque um instrumento comercial possui, via de regra, um programa de controle de difícil acesso, que não permite ao usuário adaptá-lo à uma aplicação específica. No entanto, esforços têm sido feitos no sentido de solucionar esta limitação dos instrumentos comerciais ${ }^{46}$ e, paralelamente, vários instrumentos e sistemas multicanais baseados em arranjos lineares de fotodiodos têm sido descritos na literatura, em função das necessidades específicas dos usuários ${ }^{47-54}$.

Os detectores de arranjo de diodos têm, naturalmente, amplas aplicações em determinações simultâneas sendo empregados em métodos com parada de fluxo (stopped-flow) ${ }^{55,56}$, cinéticos $^{57,58}$, derivativos ${ }^{59,60}$, quimiométricos ${ }^{61,62}$ e técnicas como cromatografia gasosa ${ }^{63}$, espectroeletroquímica ${ }^{64,65}$, espectrometria de absorção atômica ${ }^{66,67}$, de emissão atômica em plasma acoplado indutivamente ${ }^{9,68}$ e espectrografia de emissão atômica ${ }^{69}$. Ainda, os espectrofotômetros PDA têm sido também empregados em determinações onde suas propriedades multicanais não são requisitadas ${ }^{70,71}$, ocupando um espaço antes reservado apenas para as fotomultiplicadoras.

\section{REFERÊNCIAS}

1. Poppi, R. J.; Vazquez, P. A. M.; Pasquini, C.; Appl. Spectrosc. 1992, 46, 1822.

2. Perkins, W. D.; J. Chem. Ed. 1986, 63, A5.

3. Skoog, D. A.; Leary, J. J.; Principles of Instrumental Analysis, 4th. ed., Saunders College Publishing, Philadelphia, 1992; p 109-113.

4. Busch, K. W.; Busch, M. A.; Multielement Detection Systems for Spectrochemical Analysis, John Wiley, New York, 1990; cap. 15.

5. Talmi, Y.; Appl. Spectrosc. 1982, 36, 1.

6. Busch, K. W.; Busch, M. A.; Multielement Detection Systems for Spectrochemical Analysis, John Wiley, New York, 1990; p 170-176.

7. Miller, J. C.; Miller, J. N.; Statistics for Analytical Chemistry, Ellis Horwood Limited, Chichester, 1984.

8. Talmi, Y.; Anal. Chem. 1975, 47, 658A.

9. Brushwyler, K. R.; Carter, L. D.; Hieftje, G. M.; Appl. Spectrosc. 1990, 44, 1438.

10. Lobinski, R.; Marczenko, Z.; Crit. Rev. Anal. Chem. 1992, 23,55 .

11. Lázaro, F.; Ríos, A.; Luque de Castro, M. D.; Valcárcel, M.; Analusis 1986, 14, 378.

12. Busch, K. W.; Busch, M. A.; Multielement Detection Systems for Spectrochemical Analysis, John Wiley, New York, 1990; p 452-462.

13. Talmi, Y.; Simpson, R. W.; Appl. Opt. 1980, 19, 1401.

14. Jones, D. G.; Anal. Chem. 1985, 57, 1057A.

15. EG\&G Reticon, Product Description S-Series Solid State Line Scanners Data Sheet, EGG, Sunnyvale, California, 1989.

16. Bilhorn, R. B.; Epperson, P. M.; Sweedler, J. V.; Denton, M. B.; Appl. Spectrosc. 1987, 41, 1125.

17. Grossman, W. E. L.; J. Chem. Ed. 1989, 66, 697.

18. Boumans, P. W. J. M.; Brouwer, G.; Spectrochim. Acta 1972, 27B, 247.

19. Boumans, P. W. J. M.; Rumphorst, R. F.; Willemsen, L.; de Boer, F. J.; Spectrochim. Acta 1973, 28B, 227.

20. Codding, E. G.; Horlick, G.; Appl. Spectrosc. 1973, 27 , 366.

21. Horlick, G.; Codding, E. G.; Anal. Chem. 1973, 45, 1490.

22. Agnew, J. T.; Franklin, R. G.; Benn, R. E.; Bazarian, A.; J. Opt. Soc. Am. 1949, 39, 409.

23. Benn, R. E.; Foote, W. S.; Chase, C. T.; J. Opt. Soc. Am. 1949, 39, 529.

24. Busch, M. A.; Busch, K. W.; Malloy, B. B.; Talanta 1990, 37,71

25. Busch, K. W.; Busch, M. A.; Multielement Detection Systems for Spectrochemical Analysis, John Wiley, New York, 1990.

26. Dessy, R. E.; Nunn, W. G.; Titus, C. A.; J. Chromatog. Sc. 1976, 14, 195.

27. Dessy, R. E.; Reynolds, W. D.; Nunn, W. G.; Titus, C. 
A.; Moler, G. F.; J. Chromatog. 1976, 126, 347.

28. Milano, M. J.; Lam, S.; Grushka, E.; J. Chromatog. 1976, 125,315 .

29. Miller, J. C.; George, S. A.; Willis, B. G.; Science 1982, $218,241$.

30. Borman, S. A.; Anal. Chem. 1983, 55, 836A.

31. Jones, D. G.; Anal. Chem. 1985, 57, 1207A.

32. Marty, C.; Lesellier, E.; Saint-Martin, P.; Moissonnier, J. P.; Berset, C.; Analusis 1990, 18, 78.

33. Fell, A. F.; Woldemariam, T. Z.; Linley, P. A.; Jian, G.; Luque de Castro, M. D.; Valcárcel, M.; Anal. Chim. Acta 1990, 234, 89.

34. Yammamoto, A.; Matsunaga, A.; Ohto, M.; Mizukami, E.; Hayakawa, K.; Miyazaki, M.; Analyst 1995, 120, 377.

35. Anderson, L.; Anfält, T.; Granéli, A.; Strandberg, M.; Anal. Chim. Acta 1979, 109, 425.

36. Lázaro, F.; Ríos, A.; Luque de Castro, M. D.; Valcárcel, M.; Anal. Chim. Acta 1986, 179, 279.

37. León-Gonzáles, M. E.; Pérez-Arriba, L. V.; Santos-Delgado, M. J.; Polo-Díez, L. M.; Anal. Chim. Acta 1992, $258,269$.

38. Gallardo Melgarejo, A.; Cano Pavón, J. M.; Ríos Castro, A.; Anal. Chim. Acta 1990, 241, 153.

39. Gerritsen, M. J. P.; Kateman, G.; van Opstal, M. A. J.; van Bennekom, W. P.; Vandeginste, B. G. M.; Anal. Chim. Acta 1990, 241, 23.

40. Lindberg, W.; Clark, G. D.; Hanna, C. P.; Whitman, D. A.; Christian, G. D.; Ruzicka, J.; Anal. Chem. 1990, 62, 849.

41. Fernández-Band, B.; Lázaro, F.; Luque de Castro, M. D.; Valcárcel, M.; Anal. Chim. Acta 1990, 229, 177.

42. Wade, A. P.; Shiundu, P. M.; Wentzell, P. D.; Anal. Chim. Acta 1990, 237, 361.

43. Agudo, M.; Marcos, J.; Ríos, A.; Valcárcel, M.; Anal. Chim. Acta 1990, 239, 211.

44. Kowalski, B. R.; Ruzicka, J.; Christian, G. D.; Trends Anal. Chem. 1990, 9, 8.

45. Zagatto, E. A. G.; Arruda, M. A. Z.; Jacintho, A. O.; Mattos, I. L.; Anal. Chim. Acta 1990, 234, 153.

46. Shiundu, P. M.; Wade, P. A.; J. Autom. Chem. 1991, 13, 83.

47. Caceci, M. S.; Comp.Chem. 1989, 13, 33.

48. Lepla, K. C.; Horlick, G.; Appl. Spectrosc. 1989, 43, 1187.

49. Fourest, B.; David, F.; Haltier, E.; Borome, N.; Richard,
A.; Lecouturier, D.; Lalu, G.; Comp. Chem. 1991, 15, 207.

50. Naffrechoux, E.; Fachinger, C.; Suptil, J.; Anal. Chim. Acta 1992, 270, 187.

51. Gerhard, A.; Gaede, W.; Neubrand, A.; Zang, V.; van Eldik, R.; Stanitzeck, P.; Instrum. Sc. Techn. 1994, 22, 13.

52. Wingerd, M. A.; Dessy, R. E.; Appl. Spectrosc. 1990, 44, 1444.

53. Raimundo Jr., I. M.; Pasquini, C; J. Autom. Chem. 1993, $15,227$.

54. Bellato, C. R.; Rohwedder, J. J. R.; Raimundo Jr., I. M.; Pasquini, C.; J. Autom. Chem. 1996, 18, 7.

55. Gutierrez, M. C.; Gomez-Hens, A.; Pérez-Bendito, D.; Anal. Chim. Acta 1989, 225, 115.

56. Gutierrez, M. C.; Gomez-Hens, A.; Pérez-Bendito, D.; Fresenius' Z. Anal. Chem. 1989, 334, 344.

57. Peña, J. M.; Rubio, S.; Pérez-Bendito, D.; Anal. Chim. Acta 1991, 244, 81.

58. Cladera, A.; Gomes, E.; Estela, J. M.; Cerdà, V.; Cerdà, J. L .; Anal. Chim. Acta 1993, 272, 339.

59. Ares, J.; Anal. Chim. Acta 1992, 268, 135.

60. Mach, H.; Middaugh, C. R.; Lewis, R. V.; Anal. Biochem. 1992, 200, 20.

61. Bautista, R. D.; Jimenez, F.; Jimenez, A. I.; Arias, J. J.; Talanta 1993, 40, 1687.

62. Blanco, M.; Coelho, J.; Iturriaga, H.; Maspoch, S.; Bertran, E.; Fresenius' J. Anal. Chem. 1991, 340, 410.

63. Lagesson, V.; Newman, J. M.; Anal. Chem. 1989, 61, 1249.

64. Cantet, J.; Labrune, P.; Bergel, A.; Comtat, M.; Anal. Chem. 1990, 62, 1502.

65. Fultz, M. L.; Durst, R. A.; Talanta 1983, 12, 933.

66. Tittarelli, P.; Biffi, C.; J. Anal. At. Spec. 1992, 7, 409.

67. Tong, S. L.; Chin, K. S.; Spectrochim. Acta 1994, 49B, 459.

68. Brushwyler, K. R.; Furuta, N.; Hieftje, G. M.; Talanta 1990, 37, 23.

69. Brett, L.; Stahl, R. G.; Timmins, K. J.; J. Anal. At. Spec. 1989, 4, 333.

70. Shiundu, P.; Wade, A. P.; Anal. Chem. 1991, 63, 692.

71. Desmond, T. P.; Smith, R. M.; Bumfrey, T.; Anal. Proc. 1994, 31, 69 . 